\title{
Spinal cord ependymoma presenting as letm: A case report
}

\author{
Nouvakis D, Natsis KS* and Papamichalis E
}

Neurology Department, General Hospital of Serres, Greece

\begin{abstract}
Longitudinal extensive transverse myelitis (LETM) is defined as a spinal cord lesion that extends over 3 or more vertebral segments and is usually associated with NMOSD. However, intramedullary tumours may occasionally present as LETM. We present a patient with a spinal lesion over 6 vertebral segments, finally diagnosed as a spinal cord ependymoma. As with all spinal lesions, LETM must be approached with a thorough investigation and a high knowledge of its underlying causes. When NMOSD have been excluded, the clinician must consider other disorders, such as ependymomas, as the probable cause for the syndrome.
\end{abstract}

\section{Introduction}

Spinal cord ependymomas may present as a diagnostic challenge, especially when they appear as longitudinally extensive transverse myelitis (LETM), although they are the most common intramedullary tumours. LETM is a descriptive term that has been introduced in order to guide proper diagnostic work up, and is defined as a spinal cord lesion that extends over three or more vertebral segments. The majority of cases of LETM are usually associated with neuromyelitis optica spectrum disorders (NMOSD). However, intramedullary tumours may occasionally present as LETM. We present a patient with a spinal lesion over 6 vertebral segments, finally diagnosed as a spinal cord ependymoma.

\section{Case presentation}

A 60 year old man presented to the emergency department, complaining of difficulty in walking and constipation during the last 10 days which was gradually becoming worse. He also mentioned a tingling sensation in the distal lower extremities. He was a heavy smoker (50 packs /year). He quit smoking three years ago, when he was diagnosed with severe chronic obstructive pulmonary disease. No other medical conditions were mentioned and his family history was unremarkable.

Neurological examination showed decreased muscle strength (MRC 3/5) on both his lower limbs, with the proximal muscles being more seriously involved and a left Babinski sign. His lower limb tendon reflexes were symmetrically exaggerated and there was also a reduction in pain sensation below the T8 level. Upper limbs were unaffected.

Initial brain $\mathrm{CT}$ scan and routine blood tests showed no abnormalities. Cervical and thoracic MRI scan revealed an intramedullary lesion extended from T1-T6, with high signal on T2 sequences (Figure1). Additionally in T3-T4 segments the lesion showed homogenous gadolinium enhancement on $\mathrm{T} 1 \mathrm{Gd}+$ sequences (Figure 2a) and spinal swelling (Figure 2b). Brain MRI was normal. Due to the extensive length of the lesion a thorough investigation was performed. Immunological tests (ANA, ENA, anti-dsDNA, anti-Ro, anti-La, RF), serum B12, copper, ACE and anti-aquaporin 4 antibodies were reported negative. His cerebrospinal fluid (CSF) examination was normal ( 0 cells/ $\mu \mathrm{L}$, Glu: $69 \mathrm{mg} / \mathrm{dL}, \mathrm{TP}: 40,8 \mathrm{mg} / \mathrm{dL}$ ) and no infective causes for the lesion were identified. The patient received intravenus methylprednisolone (1g/day) for five days but instead of clinical improvement the patient continued to deteriorate.

An intramedullary tumour was suspected because of the unresponsiveness of the lesion to steroids and the extensive negative work up for other disorders. Ependymoma was considered more probable than astrocytoma because of its central location inside the cord and homogenous contrast enhancement. A neurosurgical consult was asked at this point, in order to plan further treatment. However, because of the serious comorbidities of the patient, he was excluded from any surgical intervention. A biopsy to establish diagnosis was not performed, due to patient's refusal. Unfortunately we are not aware of his current clinical condition, because the patient was lost in follow up.

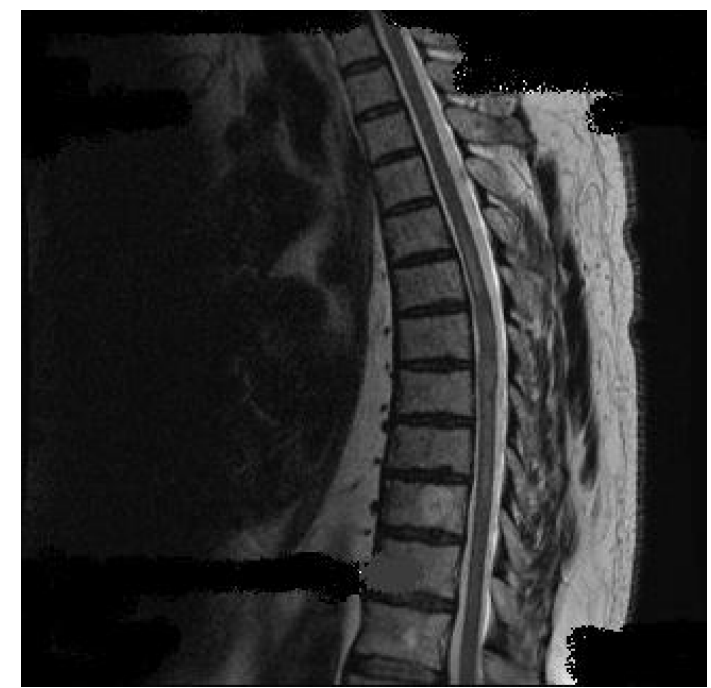

Figure 1. Intramedullary high signal lesion on $\mathrm{T} 2$ sequence extended from $\mathrm{T} 1$ to $\mathrm{T} 6$ vertebral segment.

Correspondence to: Konstantino NS, Neurology Department, General Hospital of Serres, Greece, Tel: +302321094505; E-mail: kostna1980@gmail.com

Key words: LETM, spinal ependymoma

Received: November 05, 2017; Accepted: November 27, 2017; Published: November 30, 2017 


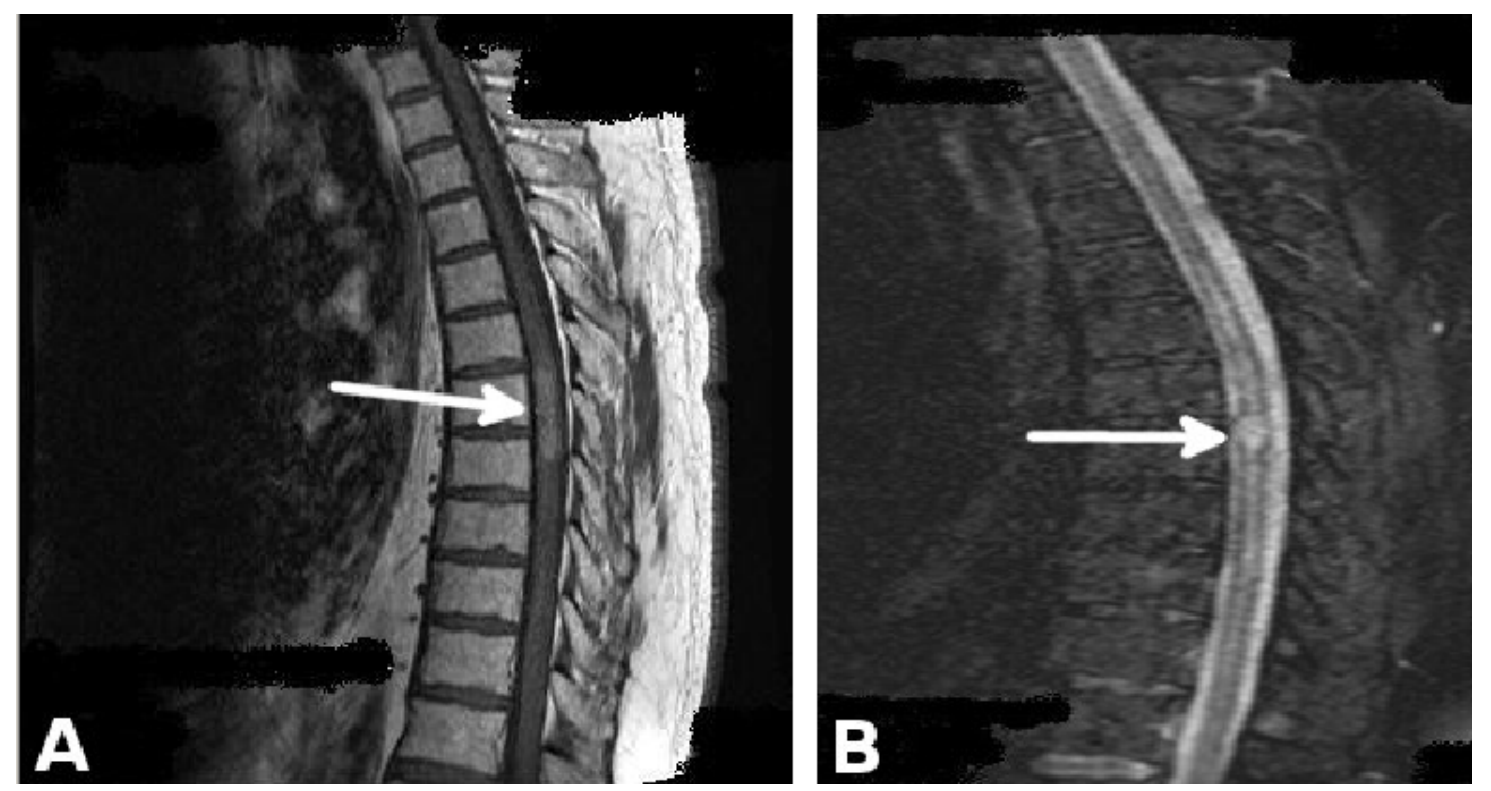

Figure 2. a. Homogenous gadolinium enhancement on T1 Gd+ sequences centrally located inside the cord on T3-T4 level; b. Spinal swelling in the area of homogenous gadolinium enhancement on $\mathrm{T} 1 \mathrm{Gd}+$

\section{Discussion}

Longitudinal extensive transverse myelitis (LETM) is defined as a spinal cord lesion that extends over 3 or more vertebral segments and is usually associated with NMOSD [1,2]. However, it can be associated with autoimmune disorders (systemic lupus, Sjogren's syndrome, Behcet disease, neurosarcoeidosis), infections (HSV, Lyme disease, HIV, syphilis), vascular malformations (dural AVF) and intramedullary tumours especially ependymoma and astrocytoma [3,4]. An extensive work up is needed, including MRI of the brain and spine, fundoscopy, CSF examination, and specific blood markers. Therapy should be tailored according to the underlying cause of the lesion.

Regarding ependymomas, their appearance in the form of LETM is rare. A recent retrospective study in found that spinal cord tumours in general account for approximately $11 \%$ of LETM [5].

Spinal cord ependymoma is the most common intramedullary tumour, followed by astrocytoma and hemangioblastoma [6-8]. Males are more commonly affected than females, and the peak incidence is at 40 years of age. It can be divided into two histological types: cellular (WHO grade II ependymoma and WHO grade III anaplastic ependymoma) and myxopapillary ependymoma (WHO grade I), which is located at the filum terminale. They can appear anywhere along the spinal cord, however the cervical cord is the most common site (44\%). An additional $23 \%$ occur within the cervical cord and extend to the thoracic cord, and $26 \%$ occur in the thoracic cord alone [6-8].

The main symptoms that are associated with spinal ependymoma are the ones that commonly appear at any lesion that causes spinal cord damage: motor weakness, sensory disturbances and autonomic involvement below the level of the lesion. However, as this is usually the case with intramedullary lesions, the sensory disturbances tend to appear first. This can be explained due to the anatomical proximity of the spinothalamic tracts to the central canal of the cord, which is the origin of ependymomas. Motor weakness and autonomic disturbances appear afterwards, once the tumour has gained considerable size.

The main diagnostic test for detecting ependymomas is MRI of the spinal cord. Ependymomas have a high signal on T2 and FLAIR sequences and in many cases present a homogenous gadolinium enhancement on $\mathrm{T} 1 \mathrm{Gd}+$ sequences and are usually centrally located inside the cord. Although necessary in detecting ependymomas, MRI cannot always distinguish them from astrocytomas. In these cases, histological confirmation via biopsy is needed [9].

In our case the negative immunological investigation, normal CSF findings and lack of clinical improvement after methypredisolone treatment, excluded autoimmune/inflammatory disorders. NMOSD was considered highly unlikely because of the negative anti-aquaporin 4 antibodies. Lymphoma was unlikely since some partial clinical and radiological improvement with corticosteroids would be expected. CSF examination failed to recognize a known infectious agent. Additionally MRI features in our case was not consistent with dural AVF. Ependymoma was considered more probable than astrocytomas in our patient because of its central location inside the cord and homogenous gadolinium enhancement.

The gold standard treatment of spinal cord ependymomas is surgical resection, with postoperative radiotherapy in cases where total exclusion was not achieved. The goal is to treat as soon as possible, in order to minimize neurological disability and improve outcome. One would expect that surgical morbidity would be less in ependymomas compared with other intramedullary tumours, since ependymomas are usually well demarcated. However, ependymomas were associated with the highest surgical morbidity rate compared with all other intramedullary tumors in a recent analysis [10]. This is the main reason that our patient was excluded from any surgical intervention. Several studies are under way in order to establish parameters associated with poor surgical outcome.

\section{Conclusions}

As with all spinal lesions, LETM must be approached with a thorough investigation and a high knowledge of its underlying causes. When NMOSD have been excluded, the clinician must consider other disorders, such as ependymomas, as the probable cause for the syndrome. Careful evaluation of the MRI findings and histological confirmation, are the basic pillars of diagnosis of these tumours. 
However one must suspect ependymomas as the cause of LETM when no other satisfactory cause has been found despite extensive work up and particularly in cases where the administration of steroids has not improved the clinical condition of the patient [11].

\section{References}

1. Lennon VA, Wingerchuk DM, Kryzer TJ, Pittock SJ, Lucchinetti CF, et al. (2004) A serum autoantibody marker of neuromyelitis optica: distinction from multiple sclerosis. Lancet 364: 2106-2112. [Crossref]

2. Wingerchuk DM, Lennon VA, Pittock SJ, Lucchinetti CF, Weinshenker BG (2006) Revised diagnostic criteria for neuromyelitis optica. Neurology 66: 1485-1489. [Crossref]

3. Bou-Haidar P, Peduto AJ, Karunaratne N (2008) Differential diagnosis of T2 hyperintense spinal cord lesions: part A. J Med Imaging Radiat Oncol 52: 535-543. [Crossref]

4. Bou-Haidar P, Peduto AJ, Karunaratne N (2009) Differential diagnosis of T2 hyperintense spinal cord lesions: part B. J Med Imaging Radiat Oncol 53: 152-159. [Crossref]
5. Contenttia CE, Hryba JP, Leguizamónb F, Di Pacea JL, Celsob J, et al. (2015) Differential diagnosis and prognosis for longitudinally extensive myelitis in Buenos Aires, Argentina. Neurologia 32: 99-105.

6. Harrop JS, Ganju A, Groff M, Bilsky M (2009) Primary intramedullary tumors of the spinal cord. Spine (Phila Pa 1976) 34(22 suppl): S69-77. [Crossref]

7. Mechtler LL, Nandigam K (2013) Spinal cord tumors: new views and future directions. Neurol Clin 31: 241-268. [Crossref]

8. Abul-Kasim K, Thurnher MM, McKeever P, Sundgren PC (2008) Intradural spinal tumors: current classification and MRI features. Neuroradiology 50: 301-314. [Crossref]

9. Do-Dai DD, Brooks MK, Goldkamp A, Erbay S, Bhadelia RA (2010) Magnetic resonance imaging of intramedullary spinal cord lesions: a pictorial review. Curr Probl Diagn Radiol 39: 160-185. [Crossref]

10. Klekamp J (2013) Treatment of intramedullary tumors: analysis of surgical morbidity and long-term results. J Neurosurg Spine 19: 12-26. [Crossref]

11. Trebst C, Raab P, Voss EV, Rommer P, Abu-Mugheisib M, et al. (2011) Longitudinal extensive transverse myelitis--it's not all neuromyelitis optica. Nat Rev Neurol 7: 68898. [Crossref]

Copyright: (C2017 Nouvakis D. This is an open-access article distributed under the terms of the Creative Commons Attribution License, which permits unrestricted use, distribution, and reproduction in any medium, provided the original author and source are credited. 